\title{
PERTURBATIONS OF NONLINEAR DIFFERENTIAL EQUATIONS
}

\author{
BY \\ R. E. FENNELL AND T. G PROCTOR
}

\begin{abstract}
Scalar and vector comparison techniques are used to study the comparative asymptotic behavior of the systems (1) $x^{\prime}=f(t, x)$ and (2) $y^{\prime}=f(t, y)+g(t, y)$. Conditions are given which allow bounds for the solutions of (2) to be obtained assuming a knowledge of the solutions of (1) and which guarantee the generalized asymptotic equivalence of (1) and (2). Examples are given.
\end{abstract}

1. Introduction. In this article we wish to compare the behavior of the solutions of a nonlinear system of differential equations and the solutions of a perturbation of this system. Brauer [2], Marlin and Struble [12] and Fennell and Proctor [4] have given previous results concerning the bounds of such solutions and concerning the asymptotic equivalence of such systems. Several other authors (see the references in [2]) have considered conditions under which various forms of the stability of a particular solution of the unperturbed system would be preserved under the perturbation. In this paper we shall not treat stability considerations.

Let $R^{+}$be the nonnegative real numbers, let $\Omega$ be a region in $R^{n}$, let $f$ and $g$ be in $C\left(R^{+} \times \Omega, R^{n}\right)$ (the continuous functions from $R^{+} \times \Omega$ into $\left.R^{n}\right)$ and be such that $f_{x}(t, x)$ exists and is continuous on $R^{+} \times \Omega$. Consider the differential equations

$$
\begin{aligned}
& x^{\prime}=f(t, x), \\
& y^{\prime}=f(t, y)+g(t, y) .
\end{aligned}
$$

Let $x(t, \gamma)$ denote the solution of (1) passing thru $\gamma$ in $\Omega$ at $t=0$, let $\Omega^{*}$ be a convex subregion of $\Omega$, and assume that for $\gamma$ in $\Omega^{*}, x(, \gamma)$ exists for $t \geq 0$. Under the conditions above the derivative matrix, $(\partial x / \partial \gamma)(t, \gamma)=\Phi(t, \gamma)$, exists and is nonsingular for $\gamma$ in $\Omega^{*}$ and $t \geq 0$ [7].

In the following sections we seek conditions on $\Omega^{*}, f, g$ which permit us to calculate bounds for the solutions of (2) assuming a knowledge of the solutions $x(t, \gamma)$ and conditions which permit us to compare the asymptotic behavior of the systems (1) and (2). We are interested in the concept of generalized asymptotic equivalence introduced in the study of perturbations of linear systems by Brauer

Received by the editors October 24, 1972.

AMS (MOS) subject classifications (1970). Primary 34D05, 34D10.

Key words and phrases. Asymptotic behavior, perturbed nonlinear differential equations, bounds, comparison theorems, generalized asymptotic equivalence. 
and Wong [1] and refined by Hallam and Heidel [5] and Ladas, Lakshmikantham and Leela [9]. The primary tools of this investigation are comparison techniques and the fact that if $z$ is a solution of

$$
z^{\prime}=G(t, z)
$$

and

$$
G(t, z)=\Phi^{-1}(t, z) g(t, x(t, z))
$$

then $y(t)=x(t, z(t))$ is a solution of (2).

We will first develop a theory using a scalar comparison technique which has been used for the perturbed linear system in [9]. Later we will show how a vector comparison technique may also be used. We have recently studied [4] the generalized asymptotic equivalence of (1) and (2) using a variation of constants formula due to Alekseev. The results below follow generally the study given by [9] for perturbation of linear systems and complements the previous study of perturbation for nonlinear equations [4].

2. Scalar comparison case. Let $\omega(t, r)$ be in $C\left(R^{+} \times R^{+}, R^{+}\right)$and be nondecreasing in $r$, and let $r(t, \delta)$ denote the maximal solution of

$$
r^{\prime}=\omega(t, r), \quad r(0)=\delta .
$$

The following theorem is an easy modification of a well-known result ([7], [11]) and will not be proved here.

Theorem A. Let $G$ be a continuous function from $R^{+} \times \Omega^{*}$, into $R^{n}$, let $|G(t, z)| \leq \omega(t,|z|)$ for $(t, z)$ in $R^{+} \times \Omega^{*}$, let $\delta>0, \gamma$ in $\Omega^{*}$ with $|\gamma| \leq \delta$ and let

$$
\Omega_{t}=\{z:|z-\gamma| \leq r(t, \delta)-\delta\}
$$

for those $t$ such that $r(t, \delta)$ is defined. If $T>0, r(T, \delta)$ is defined and $\Omega_{T} \subset \Omega^{*}$ then any solution $z$ of $(3)$, such that $z(0)=\gamma$, exists and $|z(t)-\gamma| \leq r(t, \delta)-\delta$ on $[0, T]$. Further if $r(\infty, \delta)=\lim _{t \rightarrow \infty} r(t, \delta)$ exists and $\cup_{t \geq 0} \Omega_{t} \subset \Omega^{*}$, there is a vector $\xi$ in $\Omega^{*}$ such that $\lim _{t \rightarrow \infty} z(t)=\xi$ and

$$
|z(t)-\xi| \leq r(\infty, \delta)-r(t, \delta) .
$$

The following hypothesis will be used throughout this section. For $t \geq 0, \gamma$ in $\Omega^{*}$,

$$
\left|\Phi^{-1}(t, \gamma) g(t, x(t, \gamma))\right| \leq \omega(t,|\gamma|),
$$

and for each $\sigma>0$ there is an $n \times n$ matrix $D_{0}(t)$ defined for $t>0$ such that

$$
\left|D_{\sigma}(t) \Phi(t, \gamma)\right| \leq 1,
$$

for $\gamma$ in $\Omega^{*},|\gamma| \leq \sigma, t \geq 0$. 
Theorem 1. If $\delta>0, \gamma \in \Omega^{*},|\gamma| \leq \delta$ and $T>0$ are such that $r(, \delta)$ is defined on $[0, T]$ and $\Omega_{T} \subset \Omega^{*}$, there is a solution $y$ of $(2)$ with $y(0)=\gamma$ which exists on $[0, T]$ and

$$
\left|D_{o}(t)[y(t)-x(t, \gamma)]\right| \leq r(t, \delta)-\delta
$$

for $\sigma=|\gamma|+r(T, \delta)-\delta$.

Proof. Inequality (6) implies the hypothesis of Theorem A is satisfied for the function $G$ defined by $\left(3^{\prime}\right)$. There is a solution $z$ of $(3)$ such that $z(0)=\gamma$ defined on $[0, T]$ and $|z(t)| \leq \sigma \equiv|\gamma|+r(T, \delta)-\delta$ in this interval. For $y(t)=x(t, z(t))$ we have

$$
\begin{aligned}
D_{\sigma}(t)[y(t)-x(t, \gamma)] & =D_{\sigma}(t)[x(t, z(t))-x(t, \gamma)] \\
& =\int_{0}^{1} D_{\sigma}(t) \Phi(t, s z(t)+(1-s) \gamma)(z(t)-\gamma) d s .
\end{aligned}
$$

By (7), inequality (8) holds.

Consider the special case $\omega(t, r)=\lambda(t) \phi(r)$ where $\lambda, \phi$ are continuous on $R^{+}$ and $\phi$ is nondecreasing. For a fixed $\delta>0$ we assume

$$
J(r)=\int_{\delta}^{r} \frac{d u}{\phi(u)}
$$

exists for $r \geq \delta$ and is strictly increasing. Then $J$ has an inverse $J^{-1}$ on $[0, J(\infty))$.

Corollary 1. For those $T>0$ such that $\int_{0}^{T} \lambda(s) d s$ is in the range of $J, \gamma$ in $\Omega^{*},|\gamma| \leq \delta$ and $\Omega_{T}=\left\{z:|z-\gamma| \leq J^{-1}\left(\int_{0}^{T} \lambda(s) d s\right)-\delta\right\} \subset \Omega^{*}$, there is a solution $y$ of (2) with $y(0)=\gamma$ which exists for $0 \leq t \leq T$ and

$$
\left|D_{o}(t)[y(t)-x(t, \gamma)]\right| \leq J^{-1}\left(\int_{0}^{t} \lambda(s) d s\right)-\delta
$$

where $\sigma=|\gamma|+J^{-1}\left(\int_{0}^{T} \lambda(s) d s\right)-\delta$.

We next treat the situation in which we may discuss limits as $t \rightarrow \infty$.

Theorem 2. Let $\delta>0$ be such that $r(, \delta)$ exists and is bounded on $R^{+}$, let $\gamma$ be in $\Omega^{*}$ with $|\gamma| \leq \delta$ and let $\cup_{t \geq 0} \Omega_{t} \subset \Omega^{*}$ where $\Omega_{t}$ is given by (5). There is a solution $y$ of (2) such that $y$ exists on $R^{+}, y(0)=\gamma$. Further there is a positive number $\sigma$ and there is a vector $\xi$ in $\Omega^{*}$ such that

$$
\lim _{t \rightarrow \infty} D_{o}(t)[y(t)-x(t, \xi)]=0,
$$

and

$$
\left|D_{o}(t)[y(t)-x(t, \xi)]\right| \leq r(\infty, \delta)-r(t, \delta) .
$$


Proof. We have the hypothesis for Theorem $A$ for the function $G$ defined by (3'); consequently there is a solution $z$ of $(3), z(0)=\gamma$, defined for $t \geq 0$ and a vector $\xi$ such that $z(t) \rightarrow \xi$. For $\sigma=|\gamma|+r(\infty, \delta)-\delta$ and $y(t)=x(t, z(t))$ we have

$$
D_{0}(t)[y(t)-x(t, \xi)]=\int_{0}^{1} D_{0}(t) \Phi(t, s z(t)+(1-s) \xi)(z(t)-\xi) d s
$$

which gives (10) and (11).

Corollary 2. If the hypotheses for Theorem 2 are satisfied for $\omega(t, r)=\lambda(t) \phi(r)$ and $J(r)$ given by (9) exists, inequality (11) is replaced by

$$
D_{0}(t)[y(t)-x(t, \xi)] \leq J^{-1}\left(\int_{0}^{\infty} \lambda(s) d s\right)-J^{-1}\left(\int_{0}^{t} \lambda(s) d s\right) .
$$

Theorem 2 is a statement that for certain solutions of (2) there is a corresponding solution of (1) so that the limit condition (10) holds. We consider the converse question. Under what conditions do given solutions of (1) have corresponding solutions of (2) so that (10) holds?

Theorem 3. Let $\delta \geq 0$ be such that $r(, \delta)$ exists and is bounded on $R^{+}$; let $\xi$ in $\Omega^{*}$ satisfy $|\xi|<r(\infty, \delta)$. There is a number $\sigma>0$ and a solution $y$ of (2) defined for large $t$ such that (11) holds.

Proof. We wish to show there is a solution $z$ of (3) defined for large $t$ such that $\lim _{t \rightarrow \infty} z(t)=\xi$. There is a $0<\beta \leq r(\infty, \delta)-|\xi|$ so that $\{z:|z-\xi|<\beta\} \subset \Omega^{*}$. Choose $\tau \geq 0$ so $\int_{\tau}^{\infty} \omega(t,|\xi|+\beta / 2) d t \leq \beta / 2$, let $F=\left\{z:[\tau, \infty) \rightarrow \Omega^{*}, z\right.$ continuous, $|z(t)-\xi| \leq \beta / 2\}$, and define the operator $T$ on $F$ by

$$
T z(t)=\xi-\int_{t}^{\infty} G(s, z(s)) d s, \quad \tau \leq t .
$$

It is easy to see the conditions of the Schauder-Tychonoff theorem [7] are satisfied so there is a fixed point $z$ in $F$. Consequently $y$ defined by $y(t)$ $=x(t, z(t))$ exists for $t \geq \tau$ and satisfies (2). Further inequality (11) holds for $\sigma=|\xi|+\beta / 2$.

Theorems 2 and 3 constitute a statement that systems (1) and (2) are generalized eventually asymptotically equivalent [9] in a local sense. Corresponding theorems were proved for nonlinear systems in [4]; however the hypotheses used in this article on $D_{o}(t)$ are much less restrictive. For example, here $D_{o}(t)$ is not required to be continuous or nonsingular.

It is possible to add hypotheses to Theorem 3 on the domain $\Omega^{*}$ and the comparison equations so that the solution $y$ is defined for all $t \geq 0$. For example, assume $\Omega^{*}=R^{n}$ and assume for all $0 \leq \tau<\infty, \sigma \leq \delta$ the solutions of 


$$
\rho^{\prime}=-\omega(t, \rho), \quad \rho(\tau)=\sigma
$$

are defined for $0 \leq t \leq \tau$. Since solutions $z$ of (3) are bounded on $[0, \tau]$ below the maximal solution of (12) then for $\xi$ such that $|\xi| \leq \delta$ the conclusion of Theorem 3 holds for a solution $y$ defined for all $t \geq 0$. (The condition given by (12) holds for $\omega(t, r)=\psi(t) \phi(r)$ (see [7, p. 276]).)

This modification of Theorem 3 together with Theorem 2 gives the analogue to Theorem 1 in [9] which asserts the generalized asymptotic equivalence of a linear system and its perturbations. Actually a requirement similar to that stated by equation (12) seems necessary to complete the proof of Theorems 0 and 1 in [9] because of the following example. Consider the equation (4) for $\omega(t, r)=\alpha(t) r$ $+\beta(t) r^{2}$ where $\alpha, \beta$ are to be described below. Then

$$
\begin{gathered}
r(t, \delta)=\delta \exp \int_{0}^{t} \alpha(s) d s /\left(1-\delta \int_{0}^{t} \beta(s)\left[\exp \int_{0}^{s} \alpha(u) d u\right] d s\right), \\
\rho(t, \tau, \delta)=\delta \exp -\int_{\tau}^{t} \alpha(s) d s /\left(1+\delta \int_{\tau}^{t} \beta(s) \exp \left(-\int_{\tau}^{s} \alpha(v) d v\right) d s\right) .
\end{gathered}
$$

If $\alpha$ and $\beta$ are continuous nonnegative functions approximated by

$$
\begin{aligned}
\alpha^{*}(t) & =0, & & 0 \leq t \leq \frac{1}{2}, \\
& =1, & & \frac{1}{2}<t<2, \\
& =0, & & t \geq 2, \\
\beta^{*}(t) & =1, & & 0 \leq t \leq 1, \\
& =0, & & t>1,
\end{aligned}
$$

then it is possible to choose $\delta$ so that $r(, \delta)$ is bounded on $R^{+}$and so that $\rho(t, 3 / 2, \delta)$ is not defined on $[0,3 / 2]$.

As examples of Theorems 2 and 3 consider the function $f(t, x)$ given by $f(t, x)=(x+1) \ln (x+1)$ for $x \geq 0, f(t, x)=-f(t,-x)$ for $x<0$. Then

$$
\begin{aligned}
x(t, \gamma) & =(\gamma+1)^{\exp t}-1, & & \gamma \geq 0, \\
& =1-(1-\gamma)^{\exp t}, & & \gamma<0 .
\end{aligned}
$$

If $g$ is such that $\left|\Phi^{-1}(t, z) g(t, x(t, z))\right| \leq z^{2} /(1+t)^{2}$, then we may take $D_{o}(t)$ $=e^{-t}(\sigma+1)^{1-\exp t}$ in inequality (7). For $|\gamma|<1$ there is a solution of (2) with $y(0)=\gamma$ and an $\xi$ in $R$ such that

$$
|y(t)-x(t, \xi)| \leq\left[\frac{\delta}{1-\delta}-\frac{\delta(1+t)}{1+t(1-\delta)}\right] e^{t(\sigma+1)^{(\exp t)-1}}
$$


for $\sigma=|\gamma|+\delta^{2} /(1-\delta)$. Further for $\xi$ in $R$ there is a $\sigma>0$ and a solution of (2) so that the above inequality holds. If $g$ is such that $\left|\Phi^{-1}(t, z) g(t, x(t, z))\right|$ $\leq m(t) f(t, z)$, where $m(t)$ is integrable on $R^{+}$, then with $D_{\sigma}(t)$ as given above we see

$$
|y(t)-x(t, \xi)| \leq e^{t}(1+\sigma)^{(\exp t)-1}\left[(\xi+1)-(\xi+1)^{\exp -\int_{1}^{\infty} m(s) d s}\right]
$$

which gives sufficient conditions for asymptotic equivalence in terms of $m(t)$.

Theorem 2 implies a set of solutions of (2) which are asymptotic (in a general sense) to solutions of (1). We turn now to a situation in which this set has an openness property. The corresponding theory for perturbed linear systems was developed in [5], [9].

Let $S(D)$ be the set of solutions of (2) defined on $R^{+}$such that there is a $\sigma>0$ and an $\xi$ in $\Omega^{*}$ so that (10) holds. Let $S_{0}(D)$ be that subset of functions $y$ in $S(D)$ which can be represented as $y(t)=x(t, z(t))$ for $t \geq 0$ where $z$ is a bounded solution of $(3),\left(3^{\prime}\right)$ and where there is a $\delta(y)$ such that

$$
\{\gamma:|z(t)-\gamma|<\delta(y), t \geq 0\} \subset \Omega^{*}
$$

Theorem 4. For $\gamma$ in $\Omega^{*}$, assume that the solutions of (2), $y(0)=\gamma$ are unique. Further assume the solution $z$ of $(3),\left(3^{\prime}\right)$ with $z(0)=\gamma$ depends continuously on $\gamma$. For $\delta \geq 0, \varepsilon>0$ assume there is $a \tau \geq 0$ so that the maximal solution $r(, \tau, \delta)$ of

$$
r^{\prime}=\omega(t, r), \quad r(\tau)=\delta
$$

is bounded on $t \geq \tau$ and $r(\infty, \tau, \delta)-\delta<\varepsilon$. Then for any $y$ in $S_{0}(D)$ there is an $\eta>0$ such that for every solution $y^{*}$ of (2) with $\left|y^{*}(0)-y(0)\right|<\eta$ we have $y^{*}$ is in $S_{0}(D)$.

Proof. For $y(t)=x(t, z(t))$ in $S_{0}(D), z$ is bounded for $t \geq 0$, say by $M$. Choose $\tau \geq 0$ so $r(\infty, \tau, M+\delta(y) / 2)-(M+\delta(y) / 2) \leq \delta(y) / 4$. By the continuous dependence on initial data there is an $\eta>0$ so that if $|z(0)-\gamma|<\eta, \gamma \in \Omega^{*}$, $|z(t)-z(t, \gamma)| \leq \delta(y) / 4$ for $0 \leq t \leq \tau$. Theorem $\mathrm{A}$ implies that the function $z(, \gamma)$ may be extended for $t \geq \tau$ and

$$
\begin{aligned}
|z(t, \gamma)-z(\tau, \gamma)| & =\left|\int_{\tau}^{t} G(s, z(s, \gamma)) d s\right| \\
& \leq \int_{\tau}^{\infty} \omega(s, r,(s, \tau, M+\delta(y) / 2)) d s \leq \delta(y) / 4
\end{aligned}
$$

for $t \geq \tau$. Thus $|z(t)-z(t, \gamma)| \leq 3 \delta(y) / 4$ for $t \geq 0$. Clearly there is a $\xi$ in $\Omega^{*}$ such that $z(t, \gamma) \rightarrow \xi$ and (10) holds for $y^{*}(t)=x(t, z(t, \gamma))$ and $\sigma=M+\delta(y) / 2$, and we may take $\delta\left(y^{*}\right)=\delta(y) / 4$. 
3. Vector comparison case. An analysis of the techniques used in $\$ 2$ and the work [9] shows that the crucial step is that of obtaining the asymptotic behavior of the solutions of (3). The following hypothesis will allow us to obtain such information.

Here, we use a region $\Omega^{*}=\left(a_{1}, b_{1}\right) \times \cdots \times\left(a_{n}, b_{n}\right)$ where $-\infty \leq a_{i}<b_{i}$ $\leq \infty$ for $i=1, \ldots, n$. We will work with certain critical solutions of systems of differential equations as introduced by Burton and Whyburn in [3]. Our terminology is that appearing in the text [11, pp. 21-31] by Lakshmikantham and Leela. The following theorem is straightforward.

Theorem B. Let $M, N$ and $G$ be in $C\left(R^{+} \times \Omega^{*}, R^{n}\right)$ and assume

(i) $M$ and $N$ possess a mixed quasi-monotone property for some $k, 1 \leq k \leq n$,

(ii) $M_{p}(t, z) \leq G_{p}(t, z) \leq N_{p}(t, z)$ and $M_{q}(t, z) \geq G_{q}(t, z) \geq N_{q}(t, z)$ on $R^{+} \times \Omega^{*}$,

(iii) for some $\alpha, \beta$ in $\Omega^{*}$ with $\alpha_{p} \leq \beta_{p}$ and $\alpha_{q} \geq \beta_{q}, R(t)$, the $k \max (n-k)$ min solution of $z^{\prime}=N(t, z), z(0)=\beta$, and $P(t)$, the $k \min (n-k) \max$ solution of $z^{\prime}=N(t, z), z(0)=\gamma$, exist for $t \geq 0$ and have limits $R(\infty), P(\infty)$ respectively as $t \rightarrow \infty$. Then

(a) for every $\gamma$ in $\Omega^{*}$ with $\alpha_{p} \leq \gamma_{p} \leq \beta_{p}, \alpha_{q} \geq \gamma_{q} \geq \beta_{q}$ there is a solution $z$ on $R^{+}$of (3) with $z(0)=\gamma$ and every such solution approaches a limit as $t \rightarrow \infty$;

(b) for each $\xi$ in $\Omega^{*}$ with $P_{p}(\infty)<\xi_{p}<R_{p}(\infty), P_{q}(\infty)>\xi_{q}>R_{q}(\infty)$, there is a solution $z$ of (3) defined for large $t$ such that $\lim _{t \rightarrow \infty} z(t)=\xi$.

We remark that in the proof of Theorem $B$, one obtains the following estimates on the relative size of the components of $P(t), z(t), R(t)$ and on the speed of convergence of $z(t)$ to its limiting value, $z(\infty)$,

$$
\begin{aligned}
& P_{p}(t) \leq z_{p}(t) \leq R_{p}(t), \\
& P_{q}(t) \geq z_{q}(t) \geq R_{q}(t),
\end{aligned}
$$

and

$$
\begin{aligned}
& P_{p}(\infty)-P_{p}(t) \leq z_{p}(\infty)-z_{p}(t) \leq R_{p}(\infty)-R_{p}(t), \\
& P_{q}(\infty)-P_{q}(t) \geq z_{q}(\infty)-z_{q}(t) \geq R_{q}(\infty)-R_{q}(t) .
\end{aligned}
$$

Suppose, now, that the hypotheses of Theorem B hold for $G$ defined by $\left(3^{\prime}\right)$ and for $\gamma$ in $\left\{\gamma: P_{p}(t) \leq \gamma_{p} \leq R_{p}(t), P_{q}(t) \geq \gamma_{q} \geq R_{q}(t)\right.$, for some $\left.t \geq 0\right\}$ and for $t \geq 0$ there is a matrix $D_{R P}(t)$ such that $\left|D_{R P}(t) \Phi(t, \gamma)\right| \leq 1$.

Theorem 5. Under the above hypotheses, for any $\gamma$ in $\Omega^{*}$ with $\alpha_{p} \leq \gamma_{p} \leq \beta_{p}$, $\alpha_{q} \geq \gamma_{q} \geq \beta_{q}$ there is a solution $y$ of (2) with $y(0)=\gamma$ and a solution $x(t, \xi)$ of $(1)$ such that

$$
\lim _{t \rightarrow \infty} D_{R P}(t)[y(t)-x(t, \xi)]=0 .
$$

Furthermore, if $\xi$ is in $\Omega^{*}$ and $P_{p}(\infty)<\xi_{p}<R_{p}(\infty), P_{q}(\infty)>\xi_{q}>R_{q}(\infty)$, then there is a solution $y$ of (2) defined for large $t$ such that (15) holds. 
The proof of this theorem is the same as those of Theorems 2 and 3 and will not be given here. Also using bounds (13) and (14), it is possible to obtain analogues of Theorems 1 and 4 in the present case.

4. Concluding remarks. There are important general examples of nonlinear differential equations (1) where the solution $x(t, \gamma)$ is known. The associated inequalities (6) and (7) are usually complicated and depend critically on the form of $x(t, \gamma)$. We leave open the problem of formulating requirements for allowable perturbations for these general examples and will illustrate with special cases.

Example 1. We consider the case when (1) is the Ricatti differential equation given by

$$
f(t, x)=P(t)-Q(t) x-R(t) x^{2},
$$

$P, Q, R$ are continuous for $t \geq 0$ and $u(t), t \geq 0$ is a known solution. Then

$$
\begin{aligned}
x(t, \gamma) & =u(t)+(\gamma-u(0)) e^{-h(t)} /\left(1+(\gamma-u(0)) \int_{0}^{t} R(s) e^{-h(s)} d s\right), \\
h(t) & =\int_{0}^{t}[Q(s)+2 u(s) R(s)] d s,
\end{aligned}
$$

for $\gamma$ in $R$ and $t$ nonnegative and sufficiently small that the denominator does not vanish.

To suggest the type of result which may be inferred from the theory above, consider the special case

$$
x^{\prime}+2 x-x^{2}=3-4 t^{2}
$$

where $u(t)=2 t+1$. Here equation (16) gives

$$
x(t, \gamma)=2 t+1+(\gamma-1) e^{2 t^{2}}\left[1+(1-\gamma) \int_{0}^{t} e^{2 v^{2}} d v\right]^{-1} .
$$

For $\gamma>1$ we have $x \rightarrow \infty$ in finite time and for $\gamma<1, x \rightarrow-\infty$ as $t \rightarrow \infty$. For $\gamma<1, \Phi(t, \gamma) \rightarrow 0$, hence there is a $D>0$ so that $D_{o}(t)=D$ satisfies (7). Now, consider the behavior of solutions of the perturbed equation

$$
y^{\prime}+2 y-y^{2}=3-4 t^{2}+g(t)
$$

$y(0)=\gamma$ for $|\gamma| \leq 1 / 2$. If $\omega(t, r)=a(t), \int_{0}^{\infty} a(s) d s \leq \frac{1}{4}, \Omega^{*}=\left\{z:|z| \leq \frac{3}{4}\right\}, \delta=\frac{1}{2}$ and $|g(t)| \leq 4 e^{2 t^{2}} a(t)\left[4+3 \int_{0}^{t} e^{2 v^{2}} d v\right]^{-2}$, then by Theorem 2 such a solution $y$ exists for $t \geq 0$ and there is a $\xi$ in $\Omega^{*}$ so that $x(t, \xi)-y(t) \rightarrow 0$.

In the study of solutions of

$$
y^{\prime}+Q(t) y+R(t) y^{2}=P(t)+g(t, y)
$$


in the case that the unperturbed problem has a solution $u(t)$, the transformation $R(t) y=w^{\prime} / w$ reduces the problem to the study of an equation of the type

$$
w^{\prime \prime}+Q(t) w^{\prime}+b(t) w=R(t) w q\left(t, w^{\prime} / w\right)
$$

where the unperturbed problem $w^{\prime \prime}+Q(t) w^{\prime}+P(t) w=0$ has known solutions. However the form of the perturbation does not in general fall into the class considered in previous studies [9]. The converse problem also has this feature.

Example 2. Consider the differential equation

$$
\ddot{w}+h(w)=0
$$

and the perturbed equation

$$
\ddot{u}+h(u)=k(t, u, \dot{u})
$$

where $h^{\prime}$ exists and is continuous for $|w| \leq a, h(0)=0$ and $h^{\prime}(0)>0$. By rescaling we can assume $h^{\prime}(0)=1$. Let $\varepsilon, \delta$ be positive numbers $(\delta \leq a)$ such that

(i) $w h(w)>0$ when $0<|w| \leq \delta$,

(ii) $\left|h^{\prime}(w)-1\right| \leq \varepsilon$ when $|w| \leq \delta$.

Defining $H$ by

$$
H(w)=2 \int_{0}^{w} h(u) d u, \quad|w| \leq \delta,
$$

it is well known that for $\gamma_{1}, \gamma_{2}$ satisfying

$$
0<\gamma_{2}^{2}+H\left(\gamma_{1}\right) \leq H_{1}=\min \{H(\delta), H(-\delta)\}
$$

the solution of $w(t)$ of (17) such that $w(0)=\gamma_{1}, \dot{w}(0)=\gamma_{2}$ is periodic and satisfies

$$
\dot{w}^{2}(t)+H(w(t))=\gamma_{2}^{2}+H\left(\gamma_{1}\right) .
$$

For $x=\operatorname{column}(w, \dot{w})$ the variational equation for $(17)$ is

$$
z=\left[\begin{array}{cc}
0 & 1 \\
-h^{\prime}\left(x_{1}\right) & 0
\end{array}\right] z
$$

Let $z=P v$ where

$$
P=\left[\begin{array}{cc}
1 & 1 \\
i & -i
\end{array}\right]
$$

then we have $\dot{v}=D v$, where

$$
D=\frac{1}{2} i\left[\begin{array}{cc}
h^{\prime}\left(x_{1}(t)\right)+1 & h^{\prime}\left(x_{1}(t)\right)-1 \\
-h^{\prime}\left(x_{1}(t)\right)+1 & -h^{\prime}\left(x_{1}(t)\right)-1
\end{array}\right]
$$


If we use the norm $|x|=\max \left\{\left|x_{1}\right|,\left|x_{2}\right|\right\}$ then for the logarithmic norm [Coppel, p. 41] we have

$$
\mu(D)=\frac{1}{2}\left|h^{\prime}\left(x_{1}(t)\right)-1\right|<\varepsilon .
$$

By [Coppel, p. 58] we have that

$$
|\Phi(t, \gamma)| \leq|P|\left|P^{-1}\right| e^{e t} \leq 4 e^{e t} ;
$$

thus we may choose $D_{o}(t)=\frac{1}{4} e^{-e t} I$ and $(7)$ is satisfied. A similar analysis using the adjoint of the variational equation gives $\left|\Phi^{-1}(t, \gamma)\right| \leq 4 e^{e t}$. Thus, if

$$
k\left(t, w\left(t, \gamma_{1}, \gamma_{2}\right), \dot{w}\left(t, \gamma_{1}, \gamma_{2}\right)\right) \leq \frac{1}{4} e^{-e t} \omega(t,|\gamma|)
$$

for $\gamma_{2}^{2}+H\left(\gamma_{1}\right) \leq H_{1}$, for an appropriate $\omega$ function, we have that solutions of (18) with $u(0)=\gamma_{1}, \dot{u}(0)=\gamma_{2},|\gamma|$ small are asymptotically periodic. We remark that the above analysis may sometimes be improved. For example the dynamical equations of motion for a pendulum and for a nonlinear spring are given by $h(x)=\sin x_{1}, h(x)=x+\beta x^{3}$ respectively. In each case the unperturbed system may be solved to obtain $w(t)$ in terms of elliptic functions which then gives a better estimate for $|\Phi(t, \gamma)|$.

As we mentioned in the introduction several authors have studied the asymptotic relationships between the solutions of (1) and (2). In particular a variation of constants formula was used in [4], [10], [12]. The results given in [12] concern only the case when the matrix $D_{\sigma}(t)=I$ and the solutions of (1) and (2) remain bounded; however Ladde [10] has removed the latter restriction. Fennell and Proctor [4] considered a generalized asymptotic equivalence which does not require boundedness. The type of hypotheses imposed by these studies are not in general comparable with those considered here. Kartsatos [8] has given a study of asymptotic equivalence in which no differentiability restriction is placed on $f$; however a smallness condition is imposed on the unperturbed system (1). The hypotheses used here are generalizations of those used in the study [9] of a perturbation of linear systems.

It appears that some of the results given here can be modified so that the perturbed equation (2) is a delay functional differential equation. In fact, results of this type are given for perturbations of linear systems in [6] and for perturbations of some nonlinear systems in [10].

Other examples concerning the generalized asymptotic equivalence are given in [1], [4] and [6]. A singular matrix $D_{o}(t)$ occurs in an example in [6].

\section{REFERENCES}

1. F. Brauer and J. Wong, On the asymptotic behavior of perturbed linear systems, J. Differential Equations 6 (1969), 142-153. MR 39 \#570.

2. F. Brauer, Perturbations of nonlinear systems of differential equations. IV, J. Math. Anal. Appl. 37 (1972), 214-222. MR 44 \#305. 
3. L. P. Burton and W. M. Whyburn, Minimax solutions of ordinary differential systems, Proc. Amer. Math. Soc. 3 (1952), 794-803. MR 14, 470.

4. R. Fennell and T. Proctor, On asymptotic behavior of perturbed nonlinear systems, Proc. Amer. Math. Soc. 31 (1972), 499-504. MR 44 \#4306.

5. T. Hallam and J. Heidel, The asymptotic manifold of a perturbed linear system of differential equations, Trans. Amer. Math. Soc. 149 (1970), 233-241. MR 41 \#2136.

6. T. Hallam, G. Ladas and V. Lakshmikantham, On the asymptotic behavior of functional differential equations, SIAM J. Math. Anal. 3 (1972), 58-64.

7. P. Hartman, Ordinary differential equations, Wiley, New York, 1964. MR 30 \# 1270.

8. A. Kartsatos, On the relationship between a nonlinear system and its nonlinear perturbation, J. Differential Equations 11 (1972), 582-591.

9. G. Ladas, B. Lakshmikantham and S. Leela, On the perturbability of the asymptotic manifold of a perturbed system of differential equations, Proc. Amer. Math. Soc. 27 (1971), 65-71. MR 43 \#646.

10. G. Ladde, Asymptotic equivalence and functional differential systems (to appear).

11. V. Lakshmikantham and S. Leela, Differential and integral inequalities. Vol. I, Academic Press, New York, 1969.

12. J. Marlin and R. Struble, Asymptotic equivalence of nonlinear systems, J. Differential Equations 6 (1969), 578-596, MR 40 \#5985.

Department of Mathematical Sciences, Clemson University, Clemson, South Carolina 29631 